https://doi.org/10.47660/CBR.2020.16856

\title{
PAIN AND BURN LESION: A RARE CASE OF ERYTHROMELALGIA
}

Flavio Barboza ${ }^{1, *}$, Eduarda Judith Dias Jacome Silva1 ${ }^{1}$, Ygor Augusto Silva Lima', Talles Henrique Pichinelli Maffei ${ }^{1}$, Júlia Serpa Vale ${ }^{1}$, Catharine Luísa Rocha Soares ${ }^{1}$, Heloisa Maria Lopes Scarinci ${ }^{1}$, Bruna Sayuri Tanaka ${ }^{1}$, Ian Jader Alves de Oliveira ${ }^{1}$, Raquel Gerep Pereira ${ }^{1}$, Bruna Luiza Oliveira Lima ${ }^{1}$, Nohati Rhanda Freitas dos Santos ${ }^{1}$, Thalyne Aparecida Leite de Lima ${ }^{1}$, Fernanda Roberta Loresnki ${ }^{2}$

1. Universidade Federal do Mato Grosso, Sinop (MT), Brazil. 2. Complexo Hospitalar Padre Bento, Guarulhos (SP), Brazil.

*Corresponding author: flavio.fbarboza@uol.com.br

\section{INTRODUCTION}

Erythromelalgia (EM) is a rare clinical syndrome characterized by erythema, increased skin temperature and burning pain, whose episodes manifest mainly in the distal extremities of the limbs, with the feet being more affected than the hands. According to population studies carried out in the United States, Norway and Sweden, the incidence is less than 2 per 100,000 inhabitants. It can be classified as primary / idiopathic or secondary, when it precedes or accompanies underlying causes. The latter can be divided into responsive or not to aspirin. The responsive ones are usually associated with hematological diseases such as polycythemia vera, essential thrombocytosis or other myeloproliferative disorders, whereas the nonresponsive ones are related to vascular, inflammatory or medication diseases. In this report, the objective is to describe a case of aspirin-sensitive secondary erythromelalgia.

\section{CASE REPORT}

A 46-year-old man started polyarthralgia in metacarpophalangeal tissues, wrists, elbows, knees and ankles about7years ago, in addition to a cutaneous lesion on his foot (Figs. 1 and 2). Due to high ferritin values, the patient underwent bleeding for two years in his city of origin until he was referred to the rheumatologist. On physical examination, erythematous-violet spots on the left foot were revealed, with hyperemic edges and located livedo, accompanied by intense burning pain to palpation, characteristic of neuropathic pain. Laboratory tests showed Hb: 17.7 g/dL, VG: 52.1, platelets: 540,000 mm3, ferritin: 180, ESR: 2 mm, CRP: 0.96, negative RF, negative anti-CCP and other tests within normality, including protein electrophoresis. Due to the suspicion of EM, bone marrow biopsy was requested, which indicated hypercellular erythroblastic series, hypocellular granulocytic series, moderate eosinophilia, myeloid / erythroid ratio 5:1 and hypercellular megakaryocytic series, with microforms and dysplastic forms, suggesting, thus, polycythemia vera or essential thrombocythemia. An additional dosage of erythropoietin was also performed, which presented a low serum level. In addition, a skin biopsy was performed, the result of which ruled out other causes and corroborated the clinical hypothesis of erythromelalgia. Due to the suggestion of secondary EM, the analysis of JAK2, CARL AND MPL was chosen. The patient started using acetylsalicylic acid as a therapeutic test and obtained an excellent initial response.

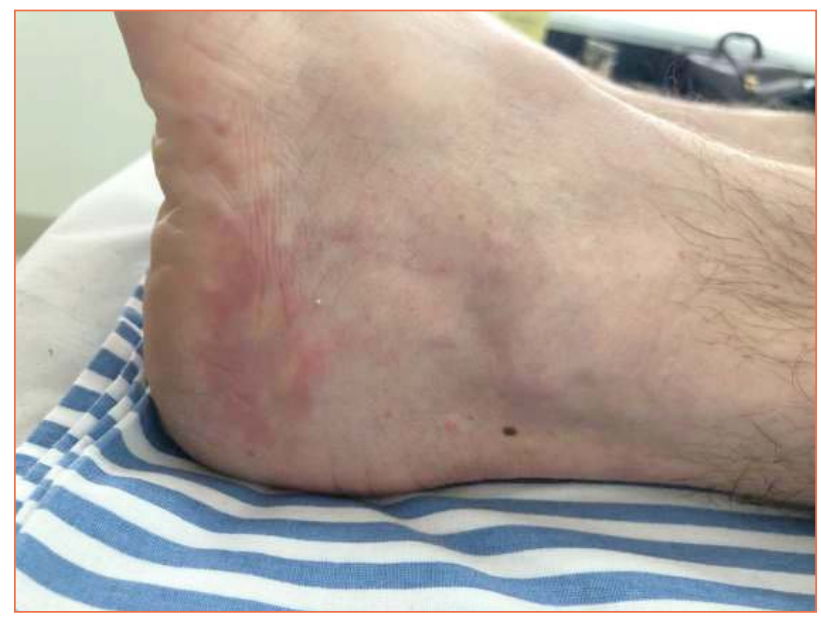

Figure 1. Erythematous-violet spots on the left foot. 


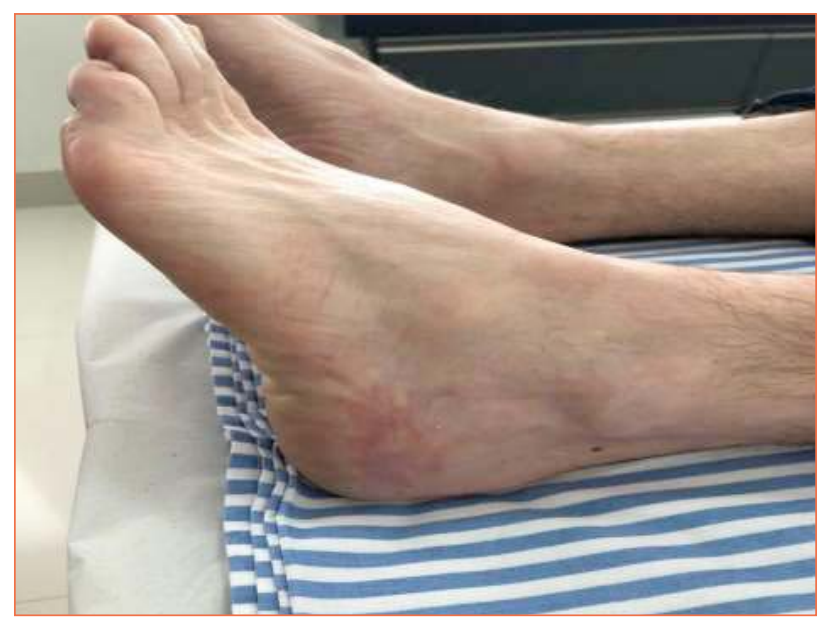

Figure 2. Hyperemic edges and located livedo.

\section{CONCLUSION}

Although it is a rare condition, it is essential that the rheumatologist not only pay attention to the clinical hypothesis of erythromelalgia, but also investigate secondary causes, among them the differentiation between polycythemia vera and essential thrombocythemia, through the analysis of the requested genes. 